\title{
Study on defects detection technique of precise optical element
}

\author{
Mi Zz ${ }^{1, *}$, Cong $\mathrm{C}^{2}$, Cheng $\mathrm{Y}^{1}$, and Zhang $\mathrm{Hm}^{3}$ \\ ${ }^{1}$ Mechanical Engineering Department, Chongqing University of Technology, 400054, China \\ ${ }^{2}$ Electrical Engineering Department, Chongqing University of Technology, 400054, China \\ ${ }^{3}$ Teaching quality monitoring and Evaluation Center, Chongqing University of Technology, 400054, China
}

\begin{abstract}
Aiming at the problems of low efficiency of traditional detection methods for surface defects of precision optical element and inconvenient detection for optical elements of different calibers, a adjustable optical element defects detecting device for large laser devices is designed. The key technical points of system composition, detection environment, illumination design and image stitching are expounded. According to the characteristics of surface defects of optical element, such as the difference of contour, gray scale, contrast and ambiguity, a classification method based on FCM is proposed. The experimental results show that the system can realize the automatic detection of surface defects, also it can effectively distinguishes micron-scale defects and has good defect recognition performance. The overall average recognition rate reached to $93.3 \%$.
\end{abstract}

\section{Introduction}

Inertial confinement fusion (ICF) is a typical case of fusion energy study that essays to initiate nuclear fusion reactions by heating and condensing a fuel target, Usually in the form of a pellet that most often contains a mixture of deuterium and tritium. ICF device is one of the important issues to solve the energy problems of humans. Nova was a high-power laser built at the Lawrence Livermore National Laboratory (LLNL, America) in 1984 that performed advanced ICF experiments until it was removed in 1999. It was the first ICF experiment to achieve the "ignition" intention ${ }^{[1]}$. The largest operational ICF experiment is the National Ignition Facility (NIF) in the America, using all of the decades accumulate experience of earlier experiments ${ }^{[2,3]}$. In addition, France's LMJ ${ }^{[4]}$, Japan's Gekko-XII and LFEX $^{[5]}$ had been put into operation. China's SG-III ${ }^{[6-8]}$ device is also progressing well. ICF driver has a very complex optical path, which contains a large number of various types and different caliber of optical elements. With the development of optical technology, the quality requirements for optical element, especially large caliber and precise optical element in the ICF drive, are becoming higher and higher. The surface defects including pits, scratches, bubble, broken edge and pollutants. The scattering effects, diffraction effects and thermal effects of defects may cause scattering, energy absorption, diffraction pattern, grinding layer destruction, laser injury etc. Those phenomena may seriously influence the beam quality, consume a lot of energy and let other element subjected to non-uniform intensity. When the beam intensity exceeds the damage threshold of element, the defects directly impact on the laser transmission and optical system quality. More serious, it may cause fatal damage to the optical system. Different Types and locations of defects in optical element, will directly affect the load capacity of ICF. So the defects detection has become an indispensable project on precision element quality Inspection.

Currently, the main defects detection method for optical element is traditional artificial visual, which takes advantage of light scattering properties in dark field illumination conditions, use optical magnifier or directly with the naked eye observation. Traditional visual method has low detection efficiency and subjectivity. In addition, the filter imaging, diffuse scattered light receiving method, the spectral analysis method is also a common means of current defects detection. These methods for the detection of smaller optical element with good results, however, ICF optics element size for large difference, which from several $\mathrm{mm}$ to several thousand $\mathrm{mm}$ away thousands of times. Standardized defection is very difficulties. In this paper, machine vision technology was used to detect surface defects of optical element, which uses non-contact and non-destructive measurement. The minimum detectable defects diameter of $4 \mu \mathrm{m}$, the maximum detectable size is $800 \times 460 \mathrm{~mm}$. Realize accurate, fast, reliable, and digital defects detection.

\section{Overall composition}

Detection device is mainly composed of a lighting module, lens, linear CCD, image acquisition card, 3-D translation stage, motion control card, optical element holder and image processing module. As shown in Figure 1. Under the illumination of a high-illuminance halogen lamp, the surface of the optical element is

\footnotetext{
* Corresponding author: lomoer@cqut.edu.cn
} 
scanned by using a three-dimensional electronic control translation device to form a target image. The target image is transmitted to the image capture card through the CameraLink protocol, and the image capture card is responsible for transmitting the image data to the image processor through the PCI bus for image storage and defects detection.

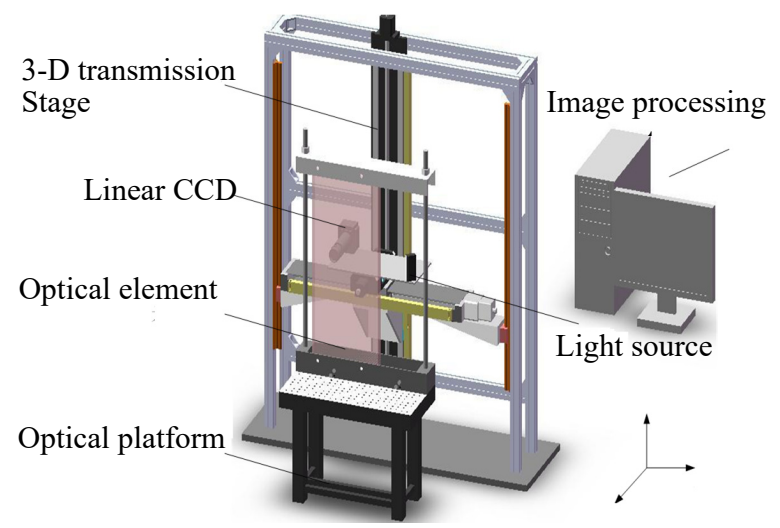

Fig. 1. 3-D Model of defects detection device

\subsection{Testing Environment}

In ICF, High-precision, large diameter and high laser damage threshold of optical element was used in amplification stage and physical diagnosis system. Optical elements range in size from $100 \mathrm{~mm} \times 100 \mathrm{~mm}$ to $800 \mathrm{~mm} \times 460 \mathrm{~mm}$, and the maximum weight of $120 \mathrm{Kg}$. Normal state of the atmosphere contains many small particles of mist. If a clean optical element under ordinary atmospheric exposure, the element of surface cleanliness can be quickly reduced. In the process of transport and detection, environmental cleanliness level should be better than 100 to reduce secondary pollution.

\subsection{Dark field illumination}

Dark field illumination principle is shown in Figure 2(a). Linear CCD was perpendicular to the optical element. Parallel light source of halogen well-proportioned illuminate to the surface with $\alpha$ degree, the most reflected light was reflected by optical element surface symmetrical to the incident light, if reflected light encounter the defects, the light will be changed into CCD. The formation of high bright defects in dark background, when the brightness difference exceeds a certain threshold, then the defects Type, area, location, quantity, shape of statistics could be determined. Compared to the bright field illumination, dark field illumination could improve the resolution and contrast of image. The contrast effect was shown in Figure 2(b).

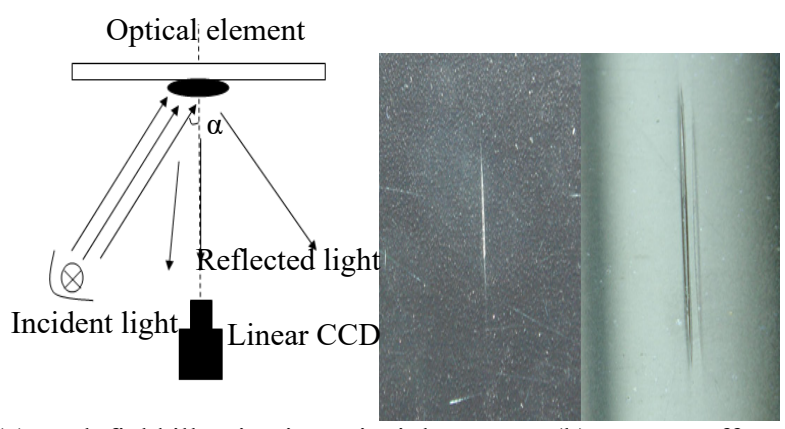

(a) Dark field illumination principle

(b) contrast effect

Fig.2. Lighting Design

\subsection{Principle of image acquisition}

In order to ensure the scanning accuracy and reduce the scanning time, the detecting device scans the element in a "snake" motion mode. The scanning schematic is shown in Figure 3. The steps are as follows:

- The linear CCD starts from the upper left edge of the optical element and scans in the positive direction of the $\mathrm{X}$ axis, and corresponds to the coordinate positions $\mathrm{A}_{11}, \mathrm{~A}_{12}, \ldots, \mathrm{A}_{1 \mathrm{n}}$.

- Move down the negative direction of the $\mathrm{Y}$ axis into the second line.

- Sub-images $A_{2 n}, A_{2(n-1)}, \ldots, A_{21}$ are sequentially scanned along the negative direction of the $\mathrm{X}$ axis.

- Move down the negative direction of the Y axis into the third line, let the device continue scanning along the positive direction of the $\mathrm{X}$ axis.

- Repeat the above steps until all sub-images of the entire optical element are obtained.

- Splicing these sub-images to have acquisition of the digital image of the optical element and storing it in the original image library.

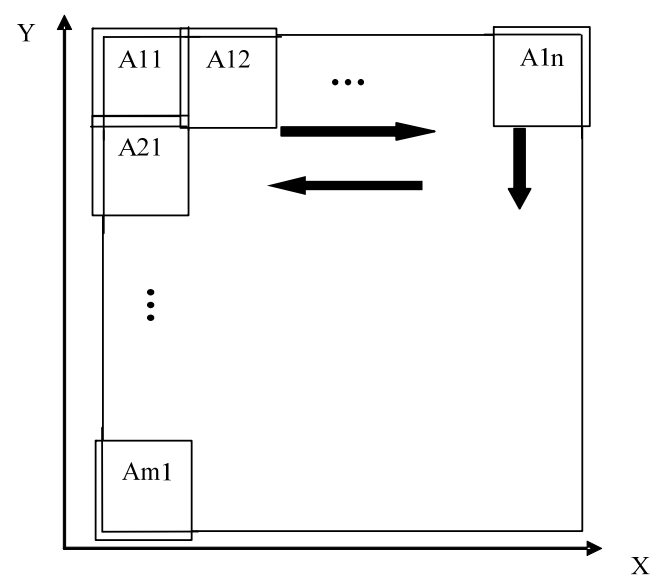

Fig. 3. Snakelike scan mode

\section{Defects detection}

\subsection{Fuzzy C-means clustering algorithm}

Fuzzy C-means clustering algorithm to $n$ vector $(j=1,2, \ldots, n)$ into $\mathrm{C}$ fuzzy set. Solving the clustering central value of every set, to let the index of dissimilarity of value function reached to minimum. Each data point 
was used membership grade to ascertain the degree of belonging to each group. The membership grade value was between 0 and 1 . With the degree of membership to determine the degree of each data point belonging to each group, membership values between 0 and 1 . After normalization, the sum of membership of a data set is equal to 1 .

$$
\sum_{i=1}^{c} u_{i j}=1, \forall j=1, \ldots, n
$$

So, FCM value function (or objective function) is a generalized form of Formula 2.

$$
J\left(U, c_{1}, \ldots, c_{c}\right)=\sum_{i=1}^{c} J_{i}=\sum_{i=1}^{c} \sum_{j}^{n} u_{i j}^{m} d_{i j}^{2}
$$

$u_{i j}$ ranged between $0,1 . c_{i}$ is the cluster center of fuzzy group I, $d i j=\left\|c_{i}-x_{i}\right\|$ is euclidean distance of the $i$-th and $j$-th cluster center between data point. $m$ is a weighted index, which the value $m \in[1, \infty)$. Construct the new the objective function to achieve the necessary condition of minimum value.

$$
\begin{aligned}
\bar{J}\left(U, c_{1}, \ldots, c_{c}, \lambda_{1}, \ldots, \lambda_{n}\right) & =J\left(U, c_{1}, \ldots, c_{c}\right)+\sum_{j=1}^{n} \lambda_{j}\left(\sum_{i=1}^{c} u_{i j}-1\right) \\
& =\sum_{i=1}^{c} \sum_{j}^{n} u_{i j}^{m} d_{i j}^{2}+\sum_{j=1}^{n} \lambda_{j}\left(\sum_{i=1}^{c} u_{i j}-1\right)
\end{aligned}
$$

$\lambda_{j}, j=1,2, \ldots, n$, is n constraints Lagrange multiplier of Formula 3. All input parameters for the derivative of formula 3 to achieve the minimum necessary conditions. Derivation of all input parameters, to let the result reached to minimum.

$$
c_{i}=\frac{\sum_{j=1}^{n} u_{i j}^{m} x_{j}}{\sum_{j=1}^{n} u_{i j}^{m}}
$$

and

$$
u_{i j}=\frac{1}{\sum_{k=1}^{c}\left(\frac{d_{i j}}{d_{k j}}\right)^{2 /(m-1)}}
$$

By the two necessary conditions above, fuzzy $C$ means clustering algorithm is a iterative process. In batch mode operation, FCM algorithm using the following steps to determine the cluster center and membership matrix $U[i]$.

Step1: The random number that range between 0 and 1 is used to initialize the membership matrix of $U$, which satisfies the constraints.

Step2: Calculation of the clustering center $c_{i}, \mathrm{i}=$ $1, \ldots, c$.

Step3: Calculation of the value function. If it is less than a certain threshold, or variable that relative to the previous value function is less than a certain threshold, algorithm stops.

Step4: Calculation of new matrix $U$, and return to step 2 .

\subsection{Feature extraction}

For defects feature extraction, defects models must be established, illustration of model was shown in Figure 4. According to the model, some features of the following were extracted:

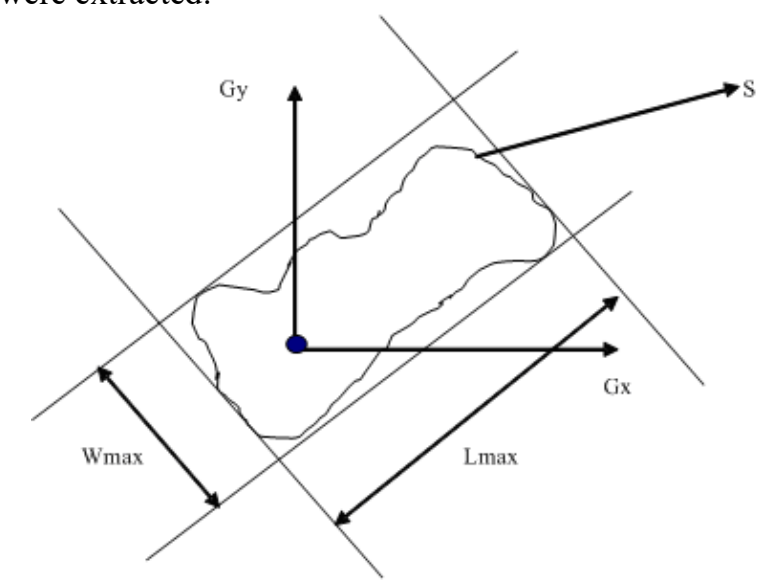

Fig.4. Schematic diagram of geometric feature extraction

a. Rectangular degree, $R_{1}=S / S_{i}$. Which is used to describe the deviation degree of the actual defects and rectangular, $Z$ has the maximum value 1 when the shape of defects is rectangular.

$b$. Elongation, $\mathrm{E}=\max \left(L_{\max }, W_{\max }\right) / \min \left(L_{\max }, W_{\max }\right)$. Which is used to describe the slender degree of the defects, $Y$ is equal to 1 when the shape was circular.

c. Roundness, $R_{2}=4 \pi S / L_{2}$. Which was used to describe the deviation degree of the actual defects and round, in the same area condition, the perimeter is the shortest when defects' edge smooth and round, and the roundness value is 1 .

d. Camber, $\mathrm{C}=\max (C C) / L, C C$ represents the chain code. Camber is used to describe the degree of smoothness of the image edges. With the chain code in the same direction up to the pixel values compared with the perimeter boundary, the $C C$ bigger, the more smooth boundary.

\subsection{Experiment and analysis of results}

The experiment adopts 3 kinds of typical surface defects in clean rooms, including scratches, pits, dust defects, each kind of defects of 40 test samples, the part of the picture shows morphological characteristics of these 3 kinds of defects, in order to facilitate observation, defects were different scales zoom, which as shown in Figure 5. 

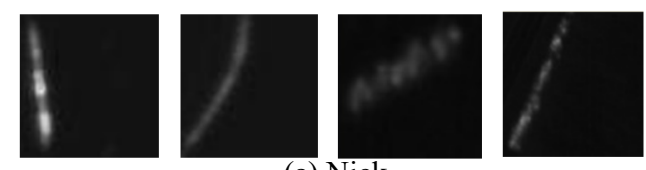

(a) Nick
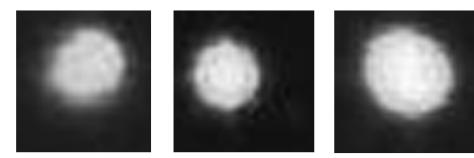

(b) Pitting
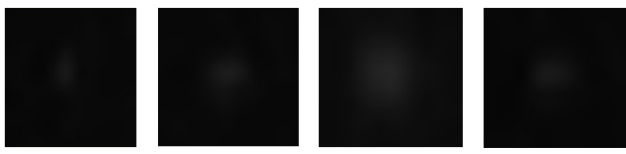

(c) Dust

Fig.5. defects in optical element

The characteristic parameters were rectangular degree, elongation, roundness, camber. Use FCM algorithm to calculate. Statistical clustering results obtained are shown in Table 1.

Table 1. Results of defects recognition.

\begin{tabular}{|c|c|c|c|c|}
\hline \multirow{2}{*}{$\begin{array}{c}\text { Defects } \\
\text { type }\end{array}$} & \multirow{2}{*}{ Quantity } & \multicolumn{3}{|c|}{ Recognition results } \\
\cline { 3 - 5 } & & Nick & Pitting & Dust \\
\hline Nick & 40 & 40 & 0 & 0 \\
\hline Pitting & 40 & 0 & 37 & 3 \\
\hline Dust & 40 & 0 & 5 & 35 \\
\hline $\begin{array}{c}\text { Recognition } \\
\text { rate (\%) }\end{array}$ & & 100 & 92.5 & 87.5 \\
\hline
\end{tabular}

From the results of the recognition results, the overall average recognition rate of $93.3 \%$. Scratches recognition rate is very high, this assay to scratch up to $100 \%$. Pitting and dust recognition rate were slightly lower. The dust recognition rate was the lowest, because when it forms a complex and sometimes difficult to distinguish between pitting and dust.

\section{Conclusion}

First of all, this paper introduces the development of ICF devices in various countries and puts forward the application background of optical element. Then the principle of image acquisition of optical element is introduced. The image matching algorithm is used to stitch the sub-aperture through the $\mathrm{X}$ and $\mathrm{Y}$ two directions of translation. In the aspect of feature extraction, for geometric characteristics and gray feature, 4 feature vectors are put forward to study the 3 kinds of typical defects. Finally, the defects were classified by FCM algorithm, and test data proved that the FCM algorithm could identify those defects very well.

\section{Acknowledgment}

This research was financially supported by the Natural Science Foundation ( KJ 1500931 ; cstc 2016 jcyjA 0447 ; cstc 2015 jcjyA 40051)

\section{References}

1. Epstein R, Regan S P, Hammel B A, et al. Applications and results of X-ray spectroscopy in implosion experiments on the National Ignition Facility[C]//AIP Conference Proceedings. AIP Publishing, 1811(1): 190004.(2017)

2. Kornblum $\mathrm{H}$ N, Kauffman $\mathrm{R}$ L, Smith $\mathrm{J}$ A. Measurement of $0.1-3-\mathrm{keV} \times$ rays from laser plasmas[J]. Review of Scientific Instruments, 57(8): 2179-2181.(1986)

3. Nagel S R, Raman K S, Huntington C M, et al. A platform for studying the Rayleigh-Taylor and Richtmyer-Meshkov instabilities in a planar geometry at high energy density at the National Ignition Facility[J]. Physics of Plasmas, 24(7): 072704.(2017)

4. Perez S, Caillaud T. The LMJ Target Diagnostics Control System Architecture[J]. (2015)

5. Shiraga H. Implosion and heating experiments of fast ignition targets by Gekko-XII and LFEX lasers[C]/CLEO: Applications and Technology. Optical Society of America, (2013)

6. Jiang S, Wang F, Ding Y, et al. Experimental Progress of Inertial Confinement Fusion Based on ShenGuang III Laser Facility in China[J]. Nuclear Fusion, (2018)

7. Wang M C, Zhu M Z, Chen G, et al. Design of Transport Beams Arrangement in Target Area of SG-III Facility[J]. Advanced Materials Research, 765: 206-209.(2013)

8. Raskar R, Velten A. Methods and apparatus for imaging of occluded objects from scattered light: U.S. Patent 20,130,100,250[P].(2013) 